\title{
BEAMFORMING AND MULTIUSER DETECTION IN CDMA SYSTEMS WITH EXTERNAL INTERFERENCES ${ }^{1}$
}

\author{
Olga Muñoz and Juan A. Fernández-Rubio \\ Departament de Teoria del Senyal i Comunicacions, UPC \\ Barcelona 08034, Spain
}

\begin{abstract}
Multiuser detection has been investigated to mitigate near-far effect in CDMA systems. Antenna arrays have shown to provide spatial diversity and cancel undesired signals. In this paper we consider the symergy of both multiuser detection and antenna arrays for the base station of a CDMA system. The receiver we proposed consists of the known multiuser decorrelator, which cancels multiple-access interferences followed by a beamformer for each user, which cancels the external interferences. This receiver adds an extra branch to the decorrelator. This additional branch, corresponding to a fictitious user with an unused code and zero power, allows to estimate the external interference signal subspace and compute a suitable bearnforming weightvector that cancels the external interferences. The receiver is also extended to the asynchronous case and all of this without any training signal or any a priori spatial information.
\end{abstract}

\section{INTRODUCTION}

Spurred by its applications in Code Division Multiple Access (CDMA), multiuser detection has been an active area of research for more than two decades $[1,2]$. As several important multipleaccess channels exhibit severe multipath fading, there is a growing interest to incorporate in some multiuser receivers explicit antenna diversity to overcome fading. In [3], Miller and Schwartz reformulate the problem of multiuser detection using spatial diversity and derive the optimum multi-element globa sequence detector for the multiuser communication. Always assuming directions and receiving array characteristics known, they derive also the spatial-temporal decorrelator, as a multisensor version of the decorrelator of Lupas and Verdú $\{1,2\}$

The unrealistic assumption of known directions and receiving array characteristics is avoided in [4], where Choi proposes another receiver which combines spatial-temporal processing for the multiuser detection problem. Choi's receiver consists of the decorrelating detector, followed by $K$ beamformers, one for each user (see figure 1). The decorrelator decouples the signal vectors of the different users. Before extracting the users' symbols, each signal vector is combined with a beamforming vector, which is no other than the spatial signature of the corresponding user. This spatial signature is calculated in a blind manner from the respective decoupled user signal vector.

The aforementioned receivers, however, fails in the presence of external interferences (interferences different from the multiple

\footnotetext{
1 This work has been partially supported by grants TIC96-0500-C10-01, TIC98-0412 and 1996SGR-00096
}

access interferences), as can be an interference from other close system or even the interference caused by another-cell user, about which the centralized receiver has no information. In [5], we consider the use of pure spatial processing to remove the distortion due to multipath fading, multiple-access and external interferences. All of this without any training signal or any a priori spatial information. The philosophy to perform the blind calculation of the suitable beamformers is similar to the one used by Choi in [4]. In [5], however, instead of the users' signals, we decouple the second order statistics of the users to compute the beamformers. Nevertheless, the performance of the receiver proposed in [5] is limited by the number of array elements. The larger the difference between the number of signals in the scenario and the number of sensors, the worse the performance. Despite of the number of external interferences with significant power it may be expected to be smaller than the number of sensors (with some frequency planning), the number of users in the cell may be expected to be larger than the number of sensors for any system with certain capacity.

In order to eliminate the degradation due to external interferences without the limitations imposed by the number of sensors, we propose a new receiver in this paper. This receiver uses multiuser detection to separate the system users and spatial processing to cancel external interferences. The new receiver combines Choi's receiver with some ideas in [5]. In order to cope with external interferences, it is necessary to increase the number of freedom degrees of Choi's receiver to estimate the interference subspace. This can be achieved by adding an extra branch to the decorrelator, that can be considered as the branch corresponding to a fictitious user with zero power.

The rest of the paper is organized as follows: Next section describes the signal model. In section 3 the proposed method is formulated. Section 4 presents some simulation results and finally in section 5 general conclusions are drawn.

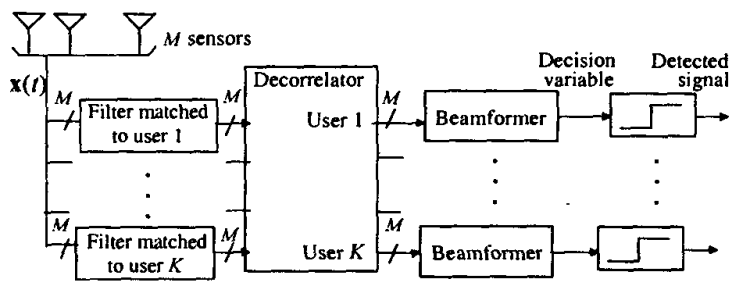

Figure 1. Choi's spatial-temporal detector [4]. 


\section{PROBLEM FORMULATION}

The system under consideration is a $K$-user asynchronous DSCDMA system using BPSK modulation and operating over a frequency non-selective channel. The baseband signal for the $k$ th user is given by

$$
b_{k}(t)=\sum_{n=0}^{N} d_{k}[n] s_{k}(t-n T)
$$

the data stream $d_{k}[n]$ is pulse amplitude modulated by the code waveform $s_{k}(t)$, with $s_{k}(t)=0$ for $t \notin\{0, T\}$ and $T$ the bit time. The received baseband signal for the multichannel case is

$$
\mathbf{x}(t)=\sum_{n=1}^{K} \sqrt{p_{k}} b_{k}(t) \mathbf{a}_{k}(t)+\mathbf{n}(t)+\mathbf{A}_{l} \mathrm{j}(t)
$$

$p_{k}, \tau_{k}$ and $a_{k}$ are respectively the transmitted power, the propagation delay and the steering vector (with dimension equal the number of sensors $M$ ) for the $k$ th user. Matrix $A_{l}$ contains the steering vectors of the interfering signals, and vector $\mathrm{j}(t)$ contains the interfering signals at time $t$ :

$$
\begin{gathered}
\mathbf{A}_{l}=\left[\begin{array}{lll}
\mathbf{a}_{K+1}(t) & \ldots & \mathbf{a}_{K+l}(t)
\end{array}\right] \\
\mathbf{j}(t)=\left[\begin{array}{lll}
j_{1}(t) & \ldots & j_{l}(t)
\end{array}\right]^{T}
\end{gathered}
$$

where $I$ the number of directional external interferences. No assumption about the temporal structure of the interferences is made.

$\mathbf{n}(t)$ is the noise vector at the array input. The noise is considered white Gaussian, uncorrelated among different sensors and with equal variance $\sigma^{2}$ for all of them. As we are assuming frequency non-selective channels, $\mathbf{a}_{k}(t)$ may be considered as the sum of $P$ coherent paths [5]. Then, we call $\mathbf{a}_{k}(t)$ the spatial signature for the $k$ th signal. This vector may be time-varying due to the combined effect of multipath and Doppler. However, in order to clarify the notation, the explicit indication of the temporal dependence of the spatial signatures will be dropped. It should be however considered implicitly. For the moment the only assumption taken about the time-varying nature of $a_{k}$ is that it is slowly varying compared to the symbol time: $\mathbf{a}_{k}(t)=\mathbf{a}_{k}(t+T)$. Later on, a more restricted assumption about the time-varying nature of $a_{k}$ will be specified.

\section{MULTIUSER AND INTERFERENCES DECORRELATION}

The receiver we propose, similar to Choi's receiver, consists of the decorrelating detector followed by $K$ beamformers. The decorrelator, however, in contrast to Choi's receiver produces $K+1$ outputs (see figure 2 ). The first one, numbered as 0 th, corresponds to a fictitious user with zero power

\subsection{Synchronous case}

For the sake of clarity, attention here will be focused first at the symbol-synchronous channel case. In synchronous transmission, each interferer user produces exactly one symbol which interferes with the desired symbol. Then, it is sufficient to consider the received signal in one symbol interval. Following [4], the received signal vector at the antenna array, $\mathbf{x}(t)$, can be expressed in a matrix form as follows:

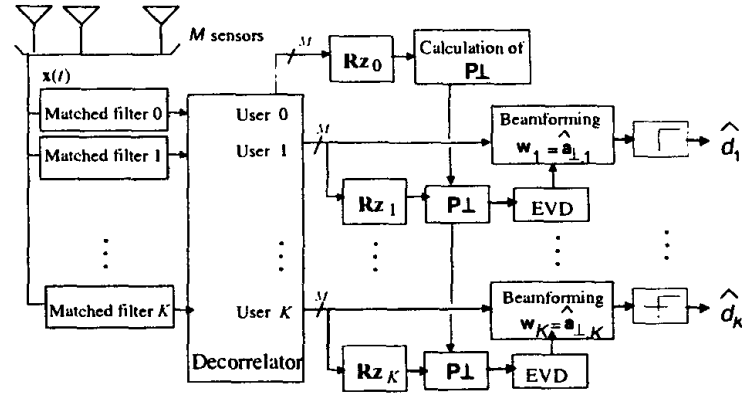

Figure 2. Proposed spatial-temporal detector.

$$
\mathbf{x}(t)=\mathbf{A P} \mathbf{P}^{1 / 2} \mathbf{D s}(t)+\mathbf{n}(t)+\mathbf{A} / \mathbf{j}(t) \quad 0 \leq t \leq T
$$

where matrix $\mathbf{P}$ is the matrix of powers, with zero in the position assigned to the fictitious user:

$$
\mathbf{P}=\operatorname{diag}\left(\left[\begin{array}{llll}
0 & p_{1} & \ldots & p_{K}
\end{array}\right]^{T}\right)
$$

matrix $\mathbf{A}$ and $\mathbf{D}$ contain respectively the spatial signatures and the symbols of the users, including the fictitious one.

$$
\begin{gathered}
\mathbf{A}=\left[\begin{array}{llll}
\mathbf{a}_{0} & \mathbf{a}_{1} & \ldots & \mathbf{a}_{K}
\end{array}\right] \\
\mathbf{D}=\operatorname{diag}\left(\left[\begin{array}{llll}
d_{0} & d_{1} & \ldots & d_{K}
\end{array}\right]^{T}\right)
\end{gathered}
$$

and vector s contains the codes of the $K$ active users along with an unused code: $\mathrm{s}_{0}(t)$.

$$
\mathbf{s}(t)=\left[\begin{array}{llll}
s_{0}(t) & s_{1}(t) & \ldots & s_{K}(t)
\end{array}\right]^{T}
$$

The values of $\mathbf{a}_{0}$ and $d_{0}$ are irrelevant since they will not take part in the subsequent calculations. Only the fictitious user code waveform will be kept.

The received signal is fed to a bank of filters matched to each spreading sequence. The outputs of the filters sampled at the bit epochs are given by the matrix:

$$
\mathbf{Y}=\int_{0}^{T} \mathbf{x}(t) \mathbf{s}^{H}(t) d t=\mathbf{A} \mathbf{P}^{1 / 2} \mathbf{D R}_{s}+\mathbf{N}+\mathbf{A}_{l} \mathbf{J}
$$

where $\mathbf{R}$, is the known code waveform correlation matrix

$$
\begin{array}{cc} 
& \mathbf{R}_{s}=\frac{1}{T} \int_{0}^{T} \mathrm{~s}\left(t \mathbf{s}^{H}(t) d t\right. \\
\text { and } & \mathbf{N}=\frac{1}{T} \int_{0}^{T} n(t) \mathrm{s}^{H}(t) d t \\
\text { with } \quad \mathbf{J}=\frac{1}{T} \int_{0}^{T} \mathrm{j}(t) \mathrm{s}^{H}(t) d t=\left[\begin{array}{llll}
\mathbf{j}_{0} & \mathbf{j}_{1} & \ldots & \mathbf{j}_{K}
\end{array}\right] \\
\mathbf{j}_{k}=\left[\begin{array}{c}
\left.\frac{1}{T} \int_{0}^{T} j_{1}(t)\right\rangle_{k}^{*}(t) d t \\
\vdots \\
\frac{1}{T} \int_{0}^{T} j_{l}(t) s_{k}^{*}(t) d t
\end{array}\right]=\left[\begin{array}{c}
j_{1, k}(t) \\
\vdots \\
j_{t, k}(t)
\end{array}\right]
\end{array}
$$


The decorrelator applies the inverse of the correlation matrix $\mathbf{R}_{s}$ to the output of the bank of matched filters, in order to decouple the users' data. The decoupled output matrix from the decorrelator is given by:

$$
\mathbf{Z}=\mathbf{Y} \mathbf{R}_{s}^{-1}=\mathbf{A} \mathbf{P}^{1 / 2} \mathbf{D}+\mathbf{N}_{z}+A_{/} \mathbf{J}_{z}
$$

where

$$
\mathbf{N}_{z}=\mathbf{N R}_{3}^{-1}
$$

$$
\mathbf{J}_{z}=\mathbf{J R}_{s}^{-1}=\left[\begin{array}{llll}
\tilde{\mathbf{J}}_{0} & \tilde{\mathbf{J}}_{1} & \ldots & \tilde{\mathbf{J}}_{K}
\end{array}\right]
$$

with $\tilde{\mathbf{J}}_{k}=\mathrm{J}\left[\mathbf{R}_{s}^{-1}\right]_{k}$, where $\left[\mathbf{R}_{s}^{-1}\right]_{k}$ denotes the $k$ th column of matrix $\mathbf{R}_{s}^{-1}$.

The $k$ th decorrelator vector output is the $k$ th column vector of matrix $\mathbf{Z}$, which can be written as:

$$
\mathbf{z}_{k}=\sqrt{p_{k}} d_{k} \mathbf{a}_{k}+\mathbf{A}_{I} \tilde{\mathbf{J}}_{k}+\mathbf{n}_{z, k}
$$

except for the first one which is

$$
\mathbf{z}_{0}=\mathbf{A}_{\zeta} \widetilde{\mathbf{J}}_{0}+\mathbf{n}_{\mathbf{z}, 0}
$$

As can be seen, each vector output $\mathbf{z}_{k}(k \neq 0)$ contains the corresponding user's information and does not have interferences from other users' signals (multiple-access interferences). From each user vector output $(k=1 \ldots K)$, an specific spatial correlation matrix is computed:

$$
\mathbf{R z}_{k}=E\left\{\mathbf{z}_{k} \mathbf{z}_{k}^{H}\right\}=p_{k} \mathbf{a}_{k} \mathbf{a}_{k}^{H}+\mathbf{A}_{I} E\left\{\tilde{\mathbf{J}}_{k} \tilde{\mathbf{J}}_{k}\right\} \mathbf{A}_{I}^{H}+\tilde{\sigma}^{2} \mathbf{I}_{M}
$$

with $\mathbf{I}_{M}$ the identity matrix with dimension $M \times M$, since the noise vector $\mathbf{n}_{i, k}$ is also spatially white [4]. For the fictitious user $(k=0)$ the correlation matrix is

$$
\mathbf{R z}_{0}=\mathbf{A}_{I} E\left\{\tilde{\mathbf{J}}_{0} \tilde{\mathbf{J}}_{0}\right\} \mathbf{A}_{I}^{H}+\tilde{\sigma}^{2} \mathbf{I}_{M}
$$

which has the same expression that $(20)$ with $p_{0}=0$.

In the absence of external interferences $\mathbf{R z}_{0}$ only has the second term corresponding to white noise. In this case the fictitious user is useless and it can be removed. Thus, the receiver becomes Choi's receiver. From (20) each $\mathbf{R} \mathbf{z}_{k}$ with $k \neq 0$ contains only one signal eigenvector. This eigenvector, which is the eigenvector associated to the main eigenvalue, is exactly the spatial signature of the corresponding user. This is the optimal array weight vector in terms of signal-to-noise ratio (SNR), since each decorrelator output is contaminated only with additive Gaussian noise.

In the presence of external interferences, however, the decorrelator outputs are contaminated with noise and interferences. Thus, the main eigenvector in each spatial correlation matrix, depending on the signal powers, may be quite different from the spatial signature of any of the involved signals. Nevertheless, it is possible to remove the information relative to the external interferences in each $\mathbf{R z}_{k}$ with $k \neq 0$, using the correlation matrix (21) of the fictitious user output vector. For this purpose, from the noise or signal subspace of $\mathbf{R} \mathbf{z}_{0}$, the projection matrix onto the interference orthogonal subspace is computed. This projection matrix is:

$$
\mathbf{P}_{\perp}=\mathbf{E}_{n, 0} \mathbf{E}_{n, 0}=\mathbf{I}_{M}-\mathbf{E}_{s, 0} \mathbf{E}_{s, 0}
$$

where $\mathbf{E}_{n, 0}$ is the noise subspace of matrix $\mathbf{R z}_{\mathbf{0}}$. Its columns are the noise subspace eigenvectors. $\mathbf{E}_{s, 0}$ is the signal subspace of matrix $\mathbf{R z}_{0}$. Its columns are the signal subspace eigenvectors.
Note that it is not necessary to calculate the spatial signatures of the interferences individually.

Next step is the projection of each matrix $\mathbf{R z}_{k}$ with $k=1 \ldots K$ onto the subspace orthogonal to the external interference subspace. As a result of the projection, each matrix $\mathbf{P}_{\perp} \mathbf{R z}_{k} \mathbf{P}_{1}(k=1 \ldots K)$ has only one signal eigenvector: $a_{\downarrow k}$. This eigenvector is the spatial signature of the corresponding user projected onto the subspace orthogonal to the external interferences, which is used as the beamformer weight vector. The new beamformers cancel the extemal interferences and obtain the best SNRs at their outputs consistent with that condition. Note that it is not necessary the knowledge of matrices $E\left\{\tilde{\mathbf{J}}_{k} \tilde{\mathbf{J}}_{k}^{H}\right\}$. In fact, they cannot be computed, but fortunately they are removed in the projection operations.

Therefore the proposed receiver cancels all kind of interferences. The multiple access interferences are removed by the decorrelator whereas the external interferences are blocked by the beamforming.

\subsection{Asynchronous case}

The extension of the proposed receiver to asynchronous systems, relays more on the decorrelating part than in the beamforming operations. The asynchronous decorrelator is fully reported in the literature $[1,2]$. Basically, we can reformulate the receiver for the asynchronous case increasing the observation interval from $0 \leq t$ $\leq T$ to $0 \leq t \leq N T+2 T$ and replacing $\mathbf{A}, \mathbf{P}, \mathbf{D}, \mathbf{s}(t)$ and $\mathbf{R}_{\mathrm{s}}$ by matrices $\mathbf{A}_{N}, \mathbf{P}_{N}, \mathbf{D}_{N}, \mathbf{s}_{\mathcal{M}}(t)$ and $\mathbf{R}_{N}$, respectively:

$$
\begin{array}{rlrl}
\mathbf{A}_{N}= & \mathbf{t}^{T} \otimes \mathbf{A}: & & \mathbf{P}_{N}=\mathbf{I}_{N} \otimes \mathbf{P} \\
\mathbf{D}_{N}= & \operatorname{diag}\left(\left[\begin{array}{llll}
{\left[d_{0}[n]\right.} & d_{1}[n] & \ldots & d_{K}[N]
\end{array}\right]^{T}\right) \\
\mathbf{s}_{N}(t)= & {\left[\begin{array}{llll}
\mathbf{s}^{T}(t) & \mathbf{s}^{T}(t-T) & \ldots & \mathbf{s}^{T}(t-N T)
\end{array}\right]^{T}} \\
& \mathbf{R}_{N}=\int_{0}^{N T+2 T} \mathbf{s}_{N}(t)_{N}^{H}(t) d t
\end{array}
$$

where $I$ an all ones $K+1$ column vector and

$$
\mathrm{s}(t-n T)=\left[\begin{array}{llll}
s_{0}\left(t-n T-\tau_{0}\right) & \ldots & s_{X}\left(t-n T-\tau_{K}\right)
\end{array}\right]^{T}
$$

Then

$$
\mathbf{Y}=\int_{0}^{N T+2 T} \mathbf{x}(t) \mathbf{s}_{N}^{H}(t) d t=\mathbf{A}_{N} \mathbf{P}_{N}^{1 / 2} \mathbf{D}_{N} \mathbf{R}_{N}+\mathbf{N}_{N}+\mathbf{A}_{J} \mathbf{J}_{N}
$$

and the vector outputs of the decorrelator are given by matrix $\mathbf{Z}$ :

$$
\mathbf{Z}=\mathbf{Y} \mathbf{R}_{N}^{-1}=\mathbf{A}_{N} \mathbf{P}_{N}^{1 / 2} \mathbf{D}_{N}+\mathbf{N}_{z}+\mathbf{A}_{I} \mathbf{J}_{z}
$$

where the first $K+1$ columns correspond to the first symbol for all the users (including the fictitious one), the next $K+1$ columns correspond to the second symbol and so on. In the previous expression

$$
\begin{aligned}
& \mathbf{N}_{\mathbf{z}}=\mathbf{N}_{N} \mathbf{R}_{N}^{-1} \\
& \mathbf{J}_{z}=\mathbf{J}_{N} \mathbf{R}_{N}^{-1}=\left[\begin{array}{llll}
\mathbf{A}_{I} \tilde{\mathbf{J}}_{0,1} & \mathbf{A}_{I} \tilde{\mathbf{J}}_{1,1} & \ldots & \mathbf{A}_{I} \tilde{\mathbf{J}}_{K, N}
\end{array}\right] \\
& \text { with } \tilde{\mathbf{J}}_{k, n}=\mathbf{J}_{N}\left[\mathbf{R}_{N}^{-1}\right]_{n k+1} \text {. From here on, the rest of the }
\end{aligned}
$$

formulation is exactly the same for the synchronous and asynchronous case. 


\section{SIMULATION RESULTS}

In order to investigate the performance of the proposed receiver an asynchronous CDMA system was simulated. The modulating signals in this system are Gold sequences with 31 chips. We consider 4 active system users and a jamming signal consisting of a BPSK signal with random chips and the same chip rate as the CDMA system signals. All the signals are received through independent Rayleigh channels. The received signal to noise ratios (SNR) are $-10 \mathrm{~dB},-5 \mathrm{~dB}, 0 \mathrm{~dB}$ and $-5 \mathrm{~dB}$ respectively for the 4 system users. The relative propagation delays for the system users are $\tau_{1}=0$ and $\tau_{2}=2 \mathrm{Tc}, \tau_{3}=5 \mathrm{Tc}$ and $\tau_{4}=7 \mathrm{Tc}$, with Tc the chip time. The array consists of $8 \lambda / 2$ linearly spaced sensors. The correlation matrix at each decorrelator output is estimated by the temporal averaging of 30 decoupled signal vectors. The channels are slowly-varying enough to assume that the spatial signatures are constant during these 30 symbols.

Figure 3 plots the output SNIR for the 1st user as a function of the interference to signal ratio for this user (ISR1). The dashed line (- -) corresponds to Choi's receiver and the solid line corresponds to the proposed receiver. The SNIR for the 1st user at the output of the classical receiver (a single filter matched to the corresponding code) is also illustrated with dashed-dotted line (-.-.). For this receiver, a single sensor Gaussian channel is considered for the four users. As can be seen, the performance of the conventional and Choi's receiver drop spectacularly as the interference power increases. This undesired effect does not occur with the receiver proposed in this paper. Note, however, that the performance of the proposed receiver for low values of interference is degraded with respect to the performance of Choi's receiver.

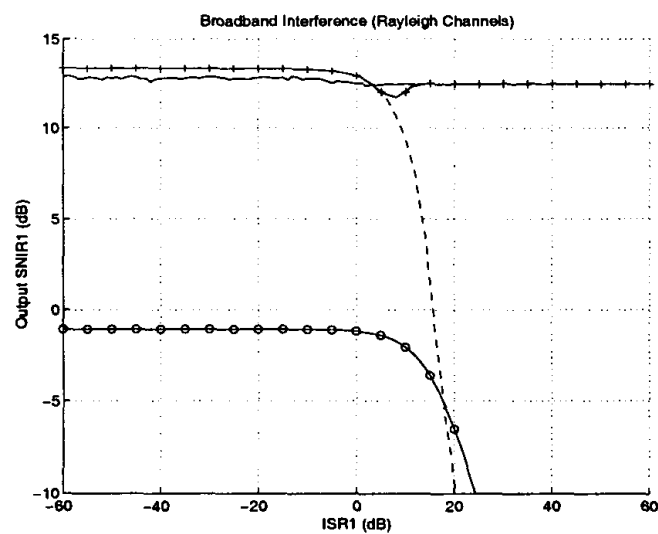

Figure 3. Output SNIR versus ISR for the 1st user. $K=4, I=1$. SNR1 $=-10 \mathrm{~dB}, \mathrm{SNR} 2=-5 \mathrm{~dB}, \mathrm{SNR} 3=0 \mathrm{~dB}$ and SNR4 $=-5 \mathrm{~dB}$ Conventional receiver (--.). Choi's receiver [4] (- - ). Proposed receiver $(\rightarrow)$. Proposed receiver with projection inhibited when the eigenvalue dispersion in matrix $\mathbf{R z}_{0}$ is greater than $2(+++)$.
This feature is due to the fact that the projection operation always provocates a noise increase. This noise increase makes that, for low values of interferences, the output noise level was higher than the noise plus interference obtained without projection. The degradation may be important depending on the considered scenario.

In order to improve the performance for the whole dynamic range of interfering power, a further refinement has been considered for the receiver. This refinement consists of blocking the projection when the eigenvalue dispersion in matrix $\mathbf{R z}_{0}$ does not exceed from a certain value, which means that the interference power is similar to the noise power. In this case the minimum value of the the eigenvalue dispersion, to avoid the projection operation, has been taken equal 2 . The resulting SNIR is shown with the solid line with plus sign $(+++)$.

\section{CONCLUSIONS}

In this paper we have considered the use of the decorrelator with antenna arrays for asynchronous CDMA systems with external interferences. The decorrelator eliminates the multiple-access interferences and the antenna array cancels those extemal interferences that the decorrelator is not able to cope with.

The beamforming in this receiver is performed easily and blindly from the decoupled signal vectors at the decorrelator output. The array weight vectors are the users' spatial signatures projected onto the subspace orthogonal to the external interferences. This subspace is obtained using an additional branch in the decorrelator, corresponding to a fictitious user with zero power. Each projected spatial signature is also obtained easily and blindly from the corresponding decoupled decorrelator output and the subspace orthogonal to the external interferences. Since no model is assumed for the steering vectors/spatial signatures the array is robust to calibration errors and the beamforming can be establish for single path or multipath channels.

A further refinement has been also considered which inhibits the projection operation for low values of the interference. This refinement avoids the noise increase due the projection, which is unnecessary if the interference power is low.

\section{REFERENCES}

[1] Lupas R. and Verdú S. "Near-Far Resistance of Multiuser Detectors in Asynchronous Channels". IEEE Transactions on Commnications, vol. 38, no. 4, April 1990, pp. 496-508.

[2] Verdú S. "Recent Progress in Multiuser Detection". Multiple Access Communications, IEEE Press, 1992.

[3] Miller S. and Schwartz S. "Integrated Spatial-Temporal Detectors for Asynchronous Gaussian Multiple-Access Channels" IEEE Transactions on Commnications, vol. 43 no. 2/3/4, Feb/March/April 1995, pp. 396-411.

[4] Choi J. "Beamforming for the Multiuser Detection with Decorrelator in Synchronous CDMA Systems. Approaches and Performance Analysis" Signal Processing, EURASIP. vol. 60 , no. 2 , July 1997 , pp. 195-211.

[5] Muñoz O. and Fernández-Rubio J. "Cancellation of External and Multiple Access Interference in CDMA Systems using Antenna Arrays". Signal Processing, EURASIP, vol. 61, September 1997, pp. 113-129. 